\title{
Monitoraggio delle emissioni odorigene in un piccolo impianto di depurazione attraverso un approccio senso-strumentale.
}

\author{
Federica Russo $^{1}$ and Domenico Giaquinto ${ }^{1}$ \\ ${ }^{1}$ Affiliation not available
}

\begin{abstract}
Le molestie olfattive indotte da impianti di trattamento di acque reflue costituiscono un vero e proprio problema ambientale. In particolare, le emissioni odorigene derivanti dall'esercizio di impianti in zone turistiche sono considerate la principale causa di disturbo notato dalla popolazione esposta. La valutazione dell'impatto indotto può essere effettuata attraverso la misurazione degli odori, utilizzando un metodo analitico o sensoriale. Quest'ultimo, legato al "sensore umano" è causa di notevole incertezza. Pertanto, nel presente articolo si vuole mostrare l'applicazione di una procedura innovativa di tipo senso-strumentale, il cui obiettivo è quello di rimuovere la componente soggettiva nella misura degli odori. I risultati ottenuti in uno SWWTP hanno dimostrato l'applicabilità di tale procedura nel monitoraggio delle emissioni odorigene ed hanno permesso l'identificazione di 39 sostanze diverse di cui quasi la metà responsabili di disturbi olfattivi.
\end{abstract}




\section{Problematiche delle emissioni odorigene e quadro normativo.}

Negli ultimi anni, l'attenzione dei cittadini verso la qualità dell'aria e l'inquinamento è aumentata in modo significativo e oggigiorno l'inquinamento da odori legato a diverse attività industriali, come impianti di trattamento e smaltimento dei rifiuti, è riconosciuto come un vero e proprio problema ambientale ${ }^{1}$. Nel caso specifico di piccoli impianti di depurazione (SWWTP) situati in zone turistiche, a causa delle grandi difficoltà legate sia alla variabilità dei carichi concentrati in pochi mesi dell'anno e sia alla semplicità dei processi applicati, è necessario limitare eventuali molestie olfattive nell' area circostante. Quest'ultime, oltre ad essere considerate la causa principale di disturbo riscontrato dalla popolazione esposta ${ }^{2}$ hanno un impatto rilevante sull'economia del turismo ${ }^{3}$. Diversi studi hanno dimostrato che i rifiuti gassosi, che causano inquinamento atmosferico sotto forma di odori, possono avere il maggiore impatto sulla popolazione in prossimità delle opere di depurazione ${ }^{4} \mathrm{e}$ in alcuni casi inducono i cittadini a presentare reclami alle autorità locali ${ }^{5}$ mostrando sintomi associati all'odore come nausea, mal di testa e mancanza di appetito ${ }^{6}$.

In tale contesto assume un ruolo di fondamentale importanza il problema del monitoraggio delle emissioni di odore. Sfortunatamente, gli odori sono difficili da misurare. La risposta di una persona a un odore è altamente soggettiva: persone diverse trovano sgradevoli odori diversi e in concentrazioni diverse. Ciò è ulteriormente complicato dal fatto che molte emissioni odorigene, comprese quelle provenienti dagli impianti di trattamento delle acque reflue, sono costituite da molti singoli componenti odorosi e l'odore complessivo di miscele complesse non può essere facilmente misurato ${ }^{2}$. Per queste ragioni, non esiste un metodo universalmente accettato per la quantificazione degli odori e la misurazione degli odori è stata spesso considerata un' arte piuttosto che una scienza ${ }^{7}$.

In ambito europeo un passo in avanti è stato fatto con la pubblicazione dello standard europeo 
EN13725 del 2003, con cui è stato identificato un metodo oggettivo per la determinazione della concentrazione di odore di un campione gassoso: l'olfattometria dinamica.

In ambito nazionale, invece, la legislazione italiana non possedeva, al 2017, alcuna indicazione normativa dedicata agli odori ma solamente criteri qualitativi e riferimenti, poco dettagliati, alla prevenzione e mitigazione delle molestie; Con l'entrata in vigore dell'articolo 272-bis nel T.U.A., introdotto dal D. Lgs. 183/2017, e stato finalmente affrontato, seppure in una fase embrionale, il tema delle emissioni odorigene industriali e agricole ${ }^{8}$. Cio nonostante, la normativa ad oggi non possiede una regolamentazione esauriente in materia di odori a livello nazionale, ma demanda alle regioni la possibilita di indicare criteri di valutazione.

I metodi presenti allo stato dell'arte nella letteratura scientifica per la misura degli odori si raggruppano in metodi sensoriali (come l'olfattometria) e metodi analitici-strumentali (come la gascromatografia/spettrometria di massa); I metodi sensoriali considerano la miscela nel suo insieme, indipendentemente dai singoli componenti mentre le tecniche analitiche, invece, permettono uno screening preliminare delle sostanze presenti, ma non consentono di avere informazioni in merito al fastidio indotto ${ }^{9}$. Esistono poi i sistemi misti che sono di recente sperimentazione e applicazione nell'ambito dell'ingegneria sanitaria ambientale ${ }^{10}$. L'obiettivo di questo studio e stato quello di studiare gli aspetti innovativi dell'utilizzo di un sistema senso-strumentale (GC/MS con porta ODP) e di valutare la relazione che vi e tra la concentrazione dei principali composti di odore e le prestazioni dell'impianto.

\section{Presentazione del caso studio}

Il caso studio preso in considerazione per l'obiettivo preposto e quello di un impianto di trattamento delle acque reflue situato nell'Universita di Stoccarda (a sud-ovest della Germania) ${ }^{11}$. I 
campioni sono stati prelevati in sette punti diversi dell'impianto durante il periodo marzo-aprile 2006. Il campionamento e stato effettuato attraverso sacche di Nalophan da 3 litri e posizionate in un contenitore rigido. Successivamente, attraverso un passaggio dei campioni in una trappola polimerica porosa, sono stati adsorbiti i composti organici per poi essere desorbiti termicamente da un gascromatografo, spurgando l'adsorbente e raccogliendo i composti in una trappola di raffreddamento. L'aria umidificata, prima di essere annusata da un esperto "annusatore di odori" e stata combinata con l'effluente GC caldo. Le analisi eseguite tramite GC-MS con un odour sniffing port (ODP) e un rilevatore a ionizzazione di fiamma (FID) sono state 23 e al fine di indagare sulla relazione che vi e tra concentrazione dei principali composti odorosi e le prestazioni dell'impianto sono stati monitorati anche BOD5, COD, PH, TD, TSS e temperatura.

I risultati hanno evidenziato la presenza di circa 39 sostanze diverse all' interno della miscela volatile del GC-MS, di cui quasi la meta sono state reputate le principali responsabili dell'odore tipico di un impianto di trattamento di acque reflue. Il disolfuro di dimetile e risultato la sostanza volatile con la soglia d'odore piu bassa e piu rilevante nell' impianto, pertanto e stato definito composto "chiave". Inoltre, il maggior contributo all'impatto degli odori e risultato proveniente direttamente dalle acque reflue grezze (50\%) e subito dopo il maggiore fastidio e stato identificato dalle attivita di trattamento dei fanghi (40\%). Per quanto concerne il BOD5 e stata mostrata una buona correlazione lineare $(65 \%)$ con la concentrazione del composto chiave. Un BOD elevato produce lo sviluppo di condizioni anaerobiche nelle acque reflue ${ }^{12}$.

Questo studio ha mostrato una tecnica innovativa che combina la soggettivita sensoriale con la precisione strumentale, nonche un metodo con cui la ricerca scientifica potrebbe essere condotta sia per caratterizzare l'impatto degli odori sia per monitorare l'efficienza degli impianti ${ }^{11}$. Ad oggi, col progredire della ricerca, i metodi applicati per la misurazione degli odori sono in aumento e in continua sperimentazione. Basti pensare ad altre tecniche sensoriali come l'ispezione sul campo, al monitoraggio strumentale attraverso i nasi elettronici e ai metodi basati sulla scienza dei 
cittadini $^{13}$, piuttosto nuovi e basate sul coinvolgimento attivo dei cittadini, per affrontare i conflitti socio-ambientali all'interno delle comunita colpite ${ }^{14}$.

\section{Discussione finale}

In conclusione, il monitoraggio degli odori e da tempo motivo di preoccupazione. Con questo caso studio, sito in Germania, si e voluto sottolineare l'importanza di una tecnica mista, sensostrumentale, nella caratterizzazione dell'impatto odorigeno in una zona turistica e la correlazione tra le concentrazioni del composto odorigeno chiave e le prestazioni dell'impianto. Un approccio integrato che combini diversi metodi e la soluzione migliore per ottenere un quadro esaustivo e completo del problema e la definizione di una buona strategia per la gestione del problema.

Cio nonostante, va precisato che ogni situazione deve essere valutata caso per caso considerando l'applicabilita ei limiti di ciascun metodo, e soprattutto le informazioni specifiche che ciascun metodo puo fornire.

\section{References}

1.Henshaw, P., Nicell, J. \& Sikdar, A. Parameters for the assessment of odour impacts on communities. Atmospheric Environment 40, 1016-1029 (2006).

2.Gostelow, P., Parsons, S. A. \& Stuetz, R. M. Odour measurements for sewage treatment works. Water Research 35, 579-597 (2001). 
3.Zarra, T. Procedures for detection and modelling of odours impact from sanitary environmental engineering plants. (University of Salerno, 2007).

4.Wilson, G. E., Schroepfer, T. W. \& Huang, J. Y. C. Atmospheric Sublayer Transport and Odor Control. Journal of the Environmental Engineering Division 106, 389-401 (1980).

5.Aatamila, M. et al.. Odour annoyance and physical symptoms among residents living near waste treatment centres. Environmental Research 111, 164-170 (2011).

6.Neutra, R., Lipscomb, J., Satin, K. \& Shusterman, D. Hypotheses to explain the higher symptom rates observed around hazardous waste sites.. Environmental Health Perspectives 94, 31-38 (1991).

7.Jiang, J. K. Odor control overview. Water environment \& technology Vol 8, Num 6, pp 55-58, (1996).

8.S.Bruzzese. Valutazione dell'impatto odorigeno prodotto da un impianto di depurazione delle acque reue sulla base delle indicazioni normative esistenti. (Politecnico di Torino, 2019).

9.S.Giuliani. Strumentazione Multisensore selettiva per il monitoraggio in continuo nel settore ambientale. (University of Salerno, 2011).

10.T.Zarra, V.Naddeo, M.Reiser \& M.Kranert. Odour emissions characterization from wastewater treatment plants by different measurement methods. Chemical Engineering Transactions Vol. 40, (2014).

11.Zarra, T., Naddeo, V., Belgiorno, V., Reiser, M. \& Kranert, M. Odour monitoring of small wastewater treatment plant located in sensitive environment. Water Science and Technology 58, 89-94 (2008).

12.Gostelow, P. \& Parsons, S. A. Sewage treatment works odour measurement. Water Science and Technology 41, 33-40 (2000). 
13.Bax, C., Sironi, S. \& Capelli, L. How Can Odors Be Measured? An Overview of Methods and Their Applications. Atmosphere 11, 92 (2020).

14.Lee, H.-D. et al.. A novel assessment of odor sources using instrumental analysis combined with resident monitoring records for an industrial area in Korea. Atmospheric Environment 74, 277-290 (2013). 
Figure Captions

Figure 1. Rielaborazione fotografica di un impianto di depurazione e della molestia olfattiva 
Figures

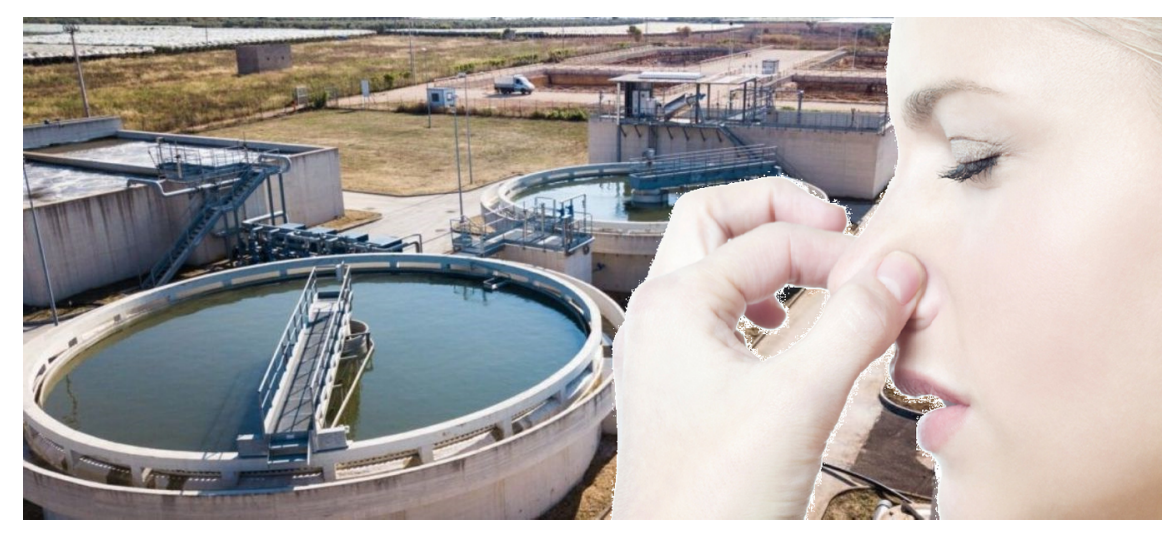

Figure 1: Rielaborazione fotografica di un impianto di depurazione e della molestia olfattiva 